\title{
Temporal analysis of pain responders and common adverse events: when do these first appear following treatment with pregabalin
}

This article was published in the following Dove Press journal: Journal of Pain Research

29 June 2015

Number of times this article has been viewed

\author{
Bruce Parsons \\ Birol Emir \\ Andrew Clair \\ Pfizer, New York, NY, USA
}

Correspondence: Bruce Parsons

Pfizer, 235 East 42nd Street, New York, NY 10017 , USA

Tel +I 2125731649

Email bruce.parsons@pfizer.com
Background: Pregabalin is an $\alpha_{2} \delta$ ligand indicated in the USA for treatment of a number of chronic pain conditions, including diabetic peripheral neuropathy, postherpetic neuralgia, pain associated with spinal cord injury, and fibromyalgia. A greater understanding of when patients first respond to treatment with pregabalin and when adverse events emerge, or worsen, could aid design of new proof-of-concept studies and help guide treatment of patients.

Methods: This was an analysis of five randomized, placebo-controlled, double-blind trials (between 8 and 16 weeks in duration) of flexible-dose pregabalin (150-600 mg/day). Individual patient data were pooled into three groups by disease condition: diabetic peripheral neuropathy or postherpetic neuralgia $(n=514)$, spinal cord injury $(n=356)$, and fibromyalgia $(n=498)$. Responders were classified as having a $\geq 30 \%$ and/or $\geq 50 \%$ reduction in mean pain score from baseline; once a patient responded, they were not scored subsequently (and were excluded from the responder analysis). The emergence of adverse events at each week was also recorded.

Results: The majority of the $30 \%$ and $50 \%$ responders emerged within the first 3-4 weeks with pregabalin, but were more uniformly distributed across the 6 weeks of the analysis with placebo. The majority of common adverse events also emerged within the first 3-4 weeks of pregabalin treatment.

Conclusion: These data suggest that the majority of pain responders and common adverse events emerge within 3-4 weeks of treatment with pregabalin. These data could advise new proof-of-concept studies and guide clinical management.

Keywords: neuropathic pain, fibromyalgia, clinical trial design

\section{Introduction}

Pregabalin is an $\alpha_{2} \delta$ ligand that is indicated in the USA for the treatment of neuropathic pain associated with diabetic peripheral neuropathy (DPN), postherpetic neuralgia (PHN), spinal cord injury (SCI), and fibromyalgia (FM), and as adjunctive therapy for partial onset seizures. ${ }^{1}$ Painful DPN and PHN are peripheral neuropathic pain syndromes affecting up to $20 \%-30 \%$ of patients with diabetes ${ }^{2}$ or herpes zoster infection. ${ }^{3,4}$ Patients with SCI typically experience some form of chronic pain, with up to $40 \%$ experiencing neuropathic pain 5 years after injury. ${ }^{5} \mathrm{FM}$ is a chronic pain disorder, with widespread pain and tenderness being the characteristic symptoms. ${ }^{6,7}$ Estimates of the incidence of FM in the adult US population range from $2 \%$ to more recent estimates of $6 \%{ }^{8,9}$

Each of these chronic pain conditions have a substantial impact on patients' healthrelated quality of life. ${ }^{10-12}$ They are also often challenging to treat, particularly so in patients who may not respond immediately to treatment due to subtherapeutic doses 
or being unable to tolerate therapy. ${ }^{13-15}$ Physicians treating these patients with pregabalin would benefit from a better understanding of when patients are most likely to respond in order to better plan their treatment strategy. Similarly, a greater understanding of when the most common adverse events (AEs) emerge or worsen could aid physicians and patients, particularly in cases where anticipation of mild AEs could facilitate improved medication adherence. Researchers designing proof-of-concept studies for new pain conditions could be guided by information on how quickly a treatment effect with pregabalin could be observed. Such information could help researchers avoid conducting unnecessarily long proof-of-concept studies. This is important, as guidelines for improving assay sensitivity in chronic pain clinical trials recommend shorter durations for proof-of-concept studies in order to maximize assay sensitivity. ${ }^{16}$ At the same time, shorter duration proof-of-concept studies would be less expensive and would reduce the exposure of patients to a potentially ineffective therapy.

This study aimed to investigate the treatment response over the first 6 weeks of clinical trials of flexible-dose pregabalin in all chronic pain conditions for which pregabalin is an approved treatment option to determine when patients first respond to treatment and when new AEs emerge or worsen. In these trials, it was decided on a weekly basis over the first 3-4 weeks of the trial if each patient's dose should be adjusted based on pain relief and AEs, after which patients completed the trial receiving their optimal dose. As the pregabalin dose was tailored to the patient's needs, this type of study design is a better representation of real-world clinical practice ${ }^{17-19}$ than a fixed-dose design, used to establish, typically for regulatory purposes, an effective dosing range for a population rather than an individual.

\section{Materials and methods Study design}

This was a pooled analysis of patient level data from five randomized, placebo-controlled, parallel-group, double-blind trials of flexible-dose pregabalin. Two trials were in patients with DPN or PHN ( $n=514)$. One, a 12-week study, consisted of 141 patients on pregabalin (150-600 mg/day in which dosage adjustments, based on efficacy and tolerability, were allowed for the first 3 weeks after which the patient remained on their optimized dose for the remainder of the trial) and 65 on placebo. ${ }^{20}$ The second was an 8 -week study consisting of 206 patients on pregabalin $(150-600 \mathrm{mg} /$ day, dosage adjustments for the first 4 weeks and optimized dose for the remainder of the trial) and 102 on placebo (ClinicalTrials.gov identifier NCT00301223). ${ }^{21}$ Two trials were in patients with neuropathic pain associated with SCI $(n=356)$. The first was a 12-week study consisting of 70 patients with SCI receiving pregabalin (150-600 mg/day, dosage adjustments for the first 3 weeks) and 67 on placebo. ${ }^{22}$ The second was a 17-week study of 112 patients with SCI receiving pregabalin (150-600 $\mathrm{mg} /$ day, dosage adjustments for the first 4 weeks) and 107 placebo (ClinicalTrials.gov identifier NCT00407745). ${ }^{23}$ Finally, there was a 15 -week study in patients with FM ( $\mathrm{n}=498)$, in which 250 patients were treated with pregabalin and 248 with placebo (ClinicalTrials.gov identifier NCT00830167). ${ }^{24}$ The FM trial included a 3-week dose-escalation/optimization phase where, after escalation from $150 \mathrm{mg}$ /day to either 300 or $450 \mathrm{mg} /$ day, the dose could be further adjusted (increased or decreased between 300 and $450 \mathrm{mg} /$ day) for an additional 2 weeks. Patients then continued on their optimized dose for a 12-week, fixed-dose treatment period. These trials were selected as their flexible dosing design is a closer representation of the optimal treatment approach, where pregabalin should be carefully titrated to the highest tolerable dose in order to maximize efficacy. ${ }^{17-19}$

All patients in these studies were $\geq 18$ years of age and had an average pain score of $\geq 4$ on the 11-point numeric rating scale, where 0 is no pain and 10 is worst possible pain, during the study screening period, with $\geq 4$ pain diaries completed. Patients with DPN were diagnosed with type 1 or type 2 diabetes mellitus and painful, symmetrical sensorimotor polyneuropathy for $\geq 6$ months. Patients with PHN had neuropathic pain for $\geq 3$ months following healing of the herpes zoster viral rash. Patients with SCI had a complete or incomplete SCI of $\geq 12$ months' duration, with chronic pain experienced continuously for $\geq 3$ months or with remissions and relapses for $\geq 6$ months. Patients with FM had a primary diagnosis of FM according to the 1990 American College of Rheumatology criteria for FM (widespread pain present for $\geq 3$ months and pain in $\geq 11$ of 18 tender points).

In one study in patients with DPN/PHN, the mean patient age was 62.2 years and the mean duration of DPN and PHN was 37.2 months and 4.7 years, respectively. ${ }^{20}$ In the second study in patients with DPN/PHN, the mean age was 60.0 years (placebo) and 60.1 years (pregabalin) and the mean duration of DPN was 3.0 years (placebo) and 2.8 years (pregabalin), and PHN was 1.3 (pregabalin and placebo) years. ${ }^{21}$ In the two studies of patients with SCI, the combined mean age was 47.2 and 47.7 years and the mean duration of pain was 107.8 and 108.1 months, for placebo and pregabalin, respectively. ${ }^{25}$ In the study of patients with 
FM, the mean age was 46.7 and 47.9 years and the mean duration since onset of FM was 62.0 and 69.6 months, for placebo and pregabalin, respectively. ${ }^{24}$

In one study of patients with DPN/PHN, the average daily pregabalin dose over the full treatment period was $372.2 \mathrm{mg} /$ day. ${ }^{20} \mathrm{In}$ patients with SCI, the average daily dose of pregabalin over both studies was $370.0 \mathrm{mg} /$ day. ${ }^{25} \mathrm{In}$ the study of patients with FM, the majority (178 of 250 [71.2\%]) received the higher dose of pregabalin $(450 \mathrm{mg} /$ day $)$ during the maintenance phase. ${ }^{24}$

\section{Pain responders}

New responders were classified as having a $\geq 30 \%$ and or $\geq 50 \%$ reduction in mean pain score from baseline. ${ }^{26}$ Once a patient responded, they were not scored subsequently and were excluded from the ongoing analysis. Mean pain score was determined as the mean score over the past 7 days as recorded by patients in a daily pain diary and measured using the 11-point numeric rating scale scored from 0 (no pain) to 10 (worst possible pain). At each week for the first 6 weeks, the numbers of new $30 \%$ and $50 \%$ responders were determined and the proportion was compared with the total number of patients remaining (ie, those who had not responded in previous weeks or were withdrawn from the trial) was calculated.

\section{Adverse events}

AEs were recorded for the full duration of each trial. In this analysis, AEs were assessed weekly where an emerging AE was scored if it appeared for the first time, or if it increased in severity. Data are shown as the percentage incidence at each week for the first 6 weeks, given that the majority of common AEs emerged within this time frame.

\section{Results \\ Efficacy}

The emergence of $30 \%$ and $50 \%$ responders was similar across disease groups with, responders typically being apparent within the first 3-4 weeks of the analysis.

In patients with DPN/PHN, the number of $30 \%$ responders peaked at week 3 with pregabalin $(15.0 \%, 22.1 \%, 26.8 \%$, and $19.1 \%$ by week for weeks $1-4$, respectively) and week 4 with placebo $(5.4 \%, 15.6 \%, 19.5 \%$, and $24.2 \%$ for weeks $1-4$, respectively, Figure 1A). In total, 231 patients $(66.8 \%)$ with pregabalin and $87(52.4 \%)$ with placebo were $30 \%$ responders after 6 weeks. The number of $50 \%$ responders peaked at 3 weeks with pregabalin $(5.5 \%, 9.1 \%, 12.9 \%$, and $10.6 \%$ for weeks $1-4$, respectively) and 4 weeks with placebo
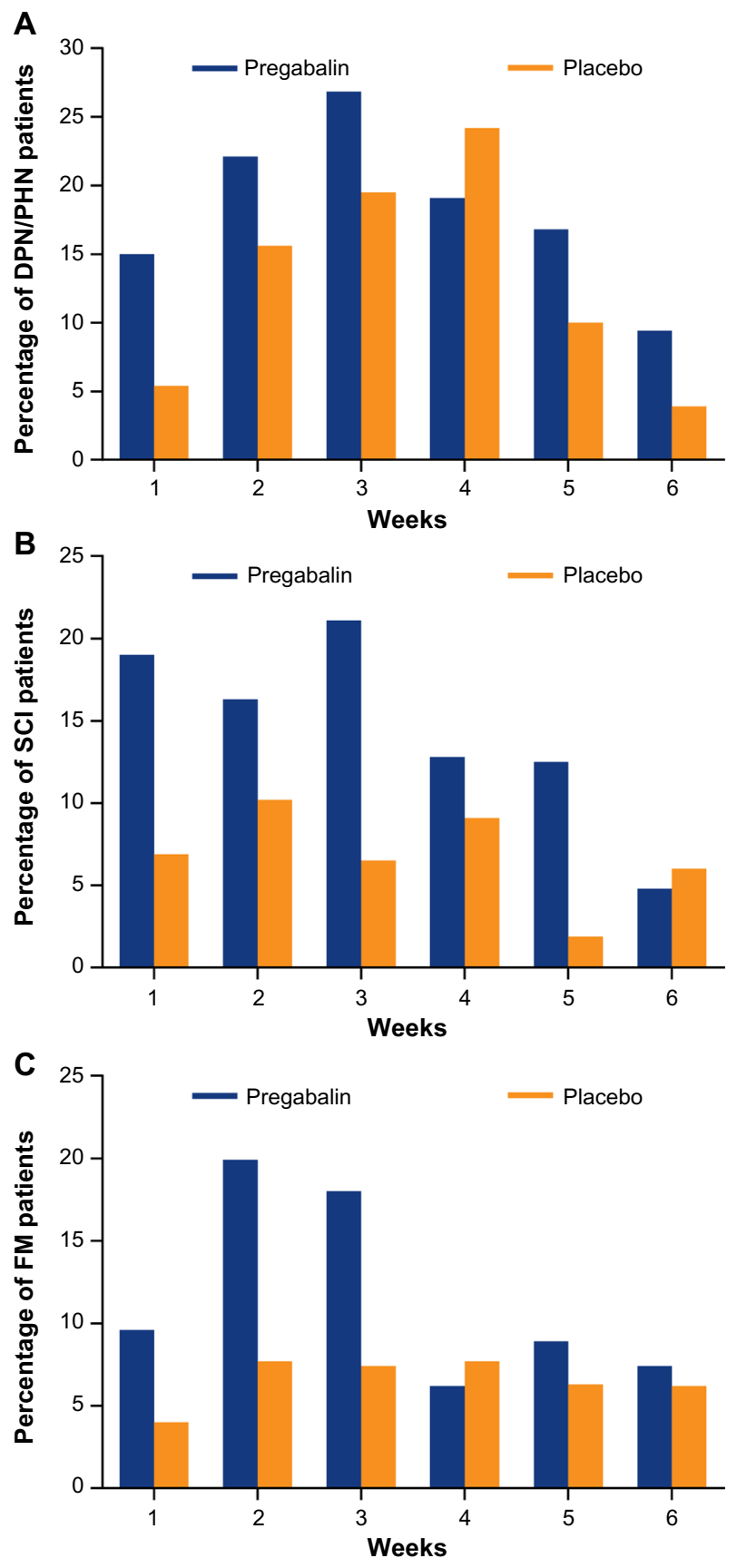

Figure I Thirty percent responders by week with pregabalin and placebo. Responders had a 30\% reduction in mean pain score. Once a patient responded, they were not scored subsequently and were excluded from the analysis. Data are shown for patients with (A) DPN/PHN, (B) SCl, and (C) FM.

Abbreviations: DPN, diabetic peripheral neuropathy; FM, fibromyalgia; PHN, postherpetic neuralgia; $\mathrm{SCl}$, spinal cord injury.

$(0.6 \%, 4.9 \%, 8.2 \%$, and $12.6 \%$ for weeks $1-4$, respectively, Figure $2 \mathrm{~A})$. In total, 144 patients (41.6\%) with pregabalin and $51(30.7 \%)$ with placebo were $50 \%$ responders in the 6-week analysis.

In patients with SCI, the majority of $30 \%$ responders emerged within the first 3 weeks of the SCI trials with pregabalin $(19.0 \%, 16.3 \%$, and $21.0 \%$ by week for weeks $1-3$, 

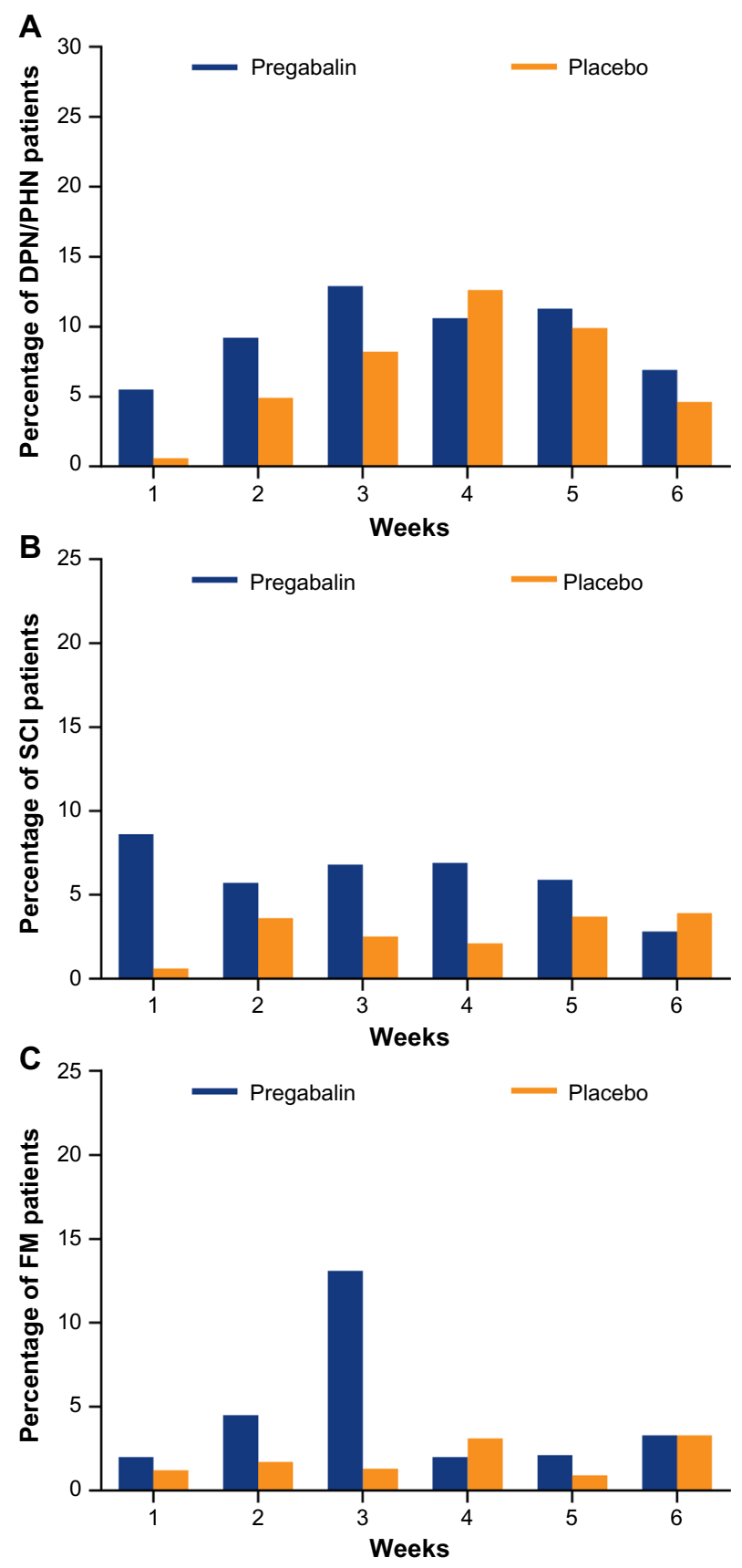

Figure 2 Fifty percent responders by week with pregabalin and placebo. Responders had a $50 \%$ reduction in mean pain score. Once a patient responded, they were not scored subsequently and were excluded from the analysis. Data are shown for patients with (A) DPN/PHN, (B) SCl, and (C) FM.

Abbreviations: DPN, diabetic peripheral neuropathy; FM, fibromyalgia; PHN, postherpetic neuralgia; $\mathrm{SCl}$, spinal cord injury.

respectively), but were more uniformly distributed across the duration of this analysis with placebo $(6.9 \%, 10.2 \%$, and $6.5 \%$ for weeks $1-3$, respectively, Figure 1B). In total, 103 patients $(59.2 \%)$ with pregabalin and $56(32.4 \%)$ with placebo were $30 \%$ responders in the 6-week analysis, with most of these emerging in the first 4 weeks. The majority of $50 \%$ responders emerged with the first $4-5$ weeks of the trials with pregabalin $(8.6 \%, 5.7 \%, 6.8 \%, 6.9 \%$, and $5.9 \%$ for weeks $1-5$, respectively) but were, again, more uniformly distributed across the duration of this analysis with placebo $(0.6 \%, 3.6 \%, 2.5 \%, 2.1 \%$, and $3.7 \%$ for weeks $1-5$, respectively, Figure $2 \mathrm{~B})$. In total, 53 patients $(30.5 \%)$ with pregabalin and $24(13.9 \%)$ with placebo were $50 \%$ responders in the 6-week analysis.

In patients with $\mathrm{FM}$, the proportion of patients who were $30 \%$ responders peaked at week 2 with pregabalin $(9.6 \%$, $19.9 \%$, and $18.0 \%$ by week for weeks $1-3$, respectively) and weeks $2-4$ with placebo $(4.0 \%, 7.7 \%, 7.4 \%$, and $7.7 \%$ for weeks $1-4$, respectively, Figure 1C). In total, 131 patients $(52.4 \%)$ with pregabalin and $80(32.3 \%)$ with placebo were $30 \%$ responders in this 6 -week analysis, most of which emerged within the first 4 weeks. The proportion of patients who were $50 \%$ responders peaked at week 3 with pregabalin (2.0\%, $4.5 \%$, and $13.1 \%$ for weeks $1-3$, respectively) and weeks $4-6(1.2 \%, 1.6 \%, 1.3 \%$, and $3.1 \%$ for weeks $1-4$, respectively) with placebo (Figure $2 \mathrm{C}$ ). In total, 60 patients $(24.0 \%)$ with pregabalin and $26(10.5 \%)$ with placebo were $50 \%$ responders in this 6 -week analysis.

\section{Emergence of adverse events}

Similar to the analysis of responders, the majority of common AEs emerged within the time frame of this analysis and, for the most part, within the first 3-4 weeks.

In patients with DPN/PHN, the most common AEs (and their incidences) with pregabalin over the entire duration of the trials were dizziness (14.4\%), peripheral edema (9.2\%), somnolence $(7.2 \%)$, and increased weight $(6.3 \%))^{20,21}$ The majority of these instances of AEs emerged within 3-4 weeks of treatment with pregabalin, with the exception of peripheral edema, where the incidence was similar at each week from weeks 2-6 (Figure 3A).

In patients with $\mathrm{SCI}$, the most common AEs (and their incidences) with pregabalin across the duration of the trials were somnolence $(35.7 \%)$, dizziness $(20.9 \%)$, dry mouth (11.0\%), fatigue (11.0\%), and peripheral edema (10.4\%). ${ }^{25}$ The majority of these AEs emerged, or worsened, within the first 4 weeks of the trials. The incidence of most AEs peaked in the first week, with the exception of peripheral edema, which peaked at week 3 (Figure 3B).

In patients with $\mathrm{FM}$, the most common AEs with pregabalin across the entire 15-week trial were somnolence, dizziness, increased weight, and constipation, with incidences of $46.4 \%, 29.6 \%, 15.6 \%$, and $14.4 \%$, respectively. ${ }^{24}$ The majority of the total incidences of these AEs emerged within the first 4 weeks in this analysis. The incidence of somnolence 

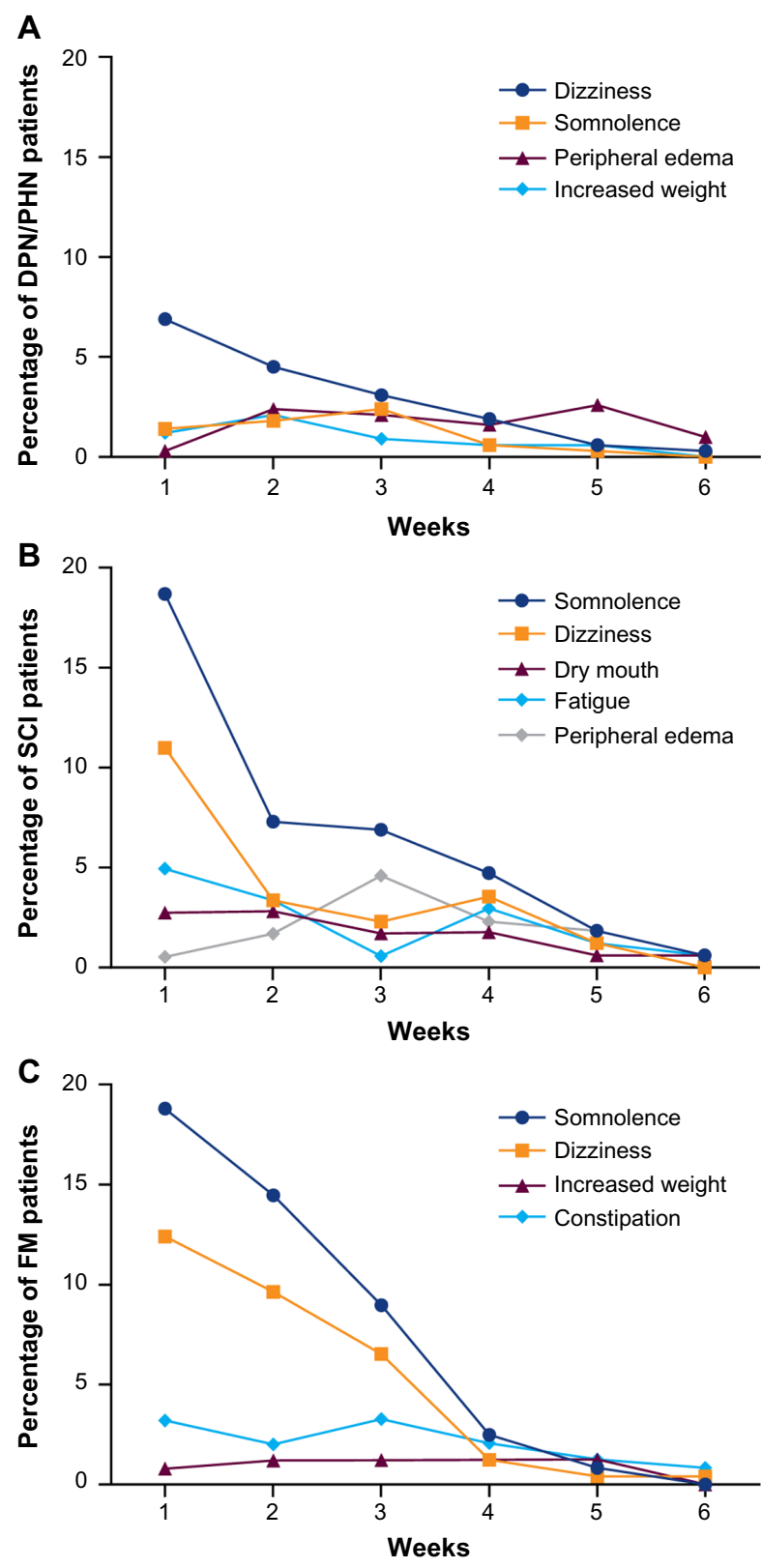

Figure 3 Incidence of common adverse events with pregabalin. Numbers of the most common adverse events, either appearing for the first time or worsening in severity, by week for the first 6 weeks. Data are shown for patients with (A) DPN/ $\mathrm{PHN},(\mathbf{B}) \mathrm{SCl}$, and (C) FM.

Abbreviations: DPN, diabetic peripheral neuropathy; FM, fibromyalgia; PHN, postherpetic neuralgia; $\mathrm{SCl}$, spinal cord injury.

and dizziness peaked at week 1 , with a lower incidence at weeks 2 and 3, by which point the majority of incidences had emerged (Figure 3C). Constipation occurred at a similar incidence for each of the first 4 weeks, after which the incidence reduced.

\section{Discussion}

This analysis of five clinical trials of flexible-dose pregabalin for the treatment of those chronic pain conditions for which pregabalin is an approved therapy revealed that the majority of patients respond to treatment within the first 3-4 weeks of treatment. Similarly, the majority of the most common AEs in these trials had emerged within the first 3-4 weeks.

The total proportion of responders to emerge within the first 6 weeks in this analysis was similar to the proportion of responders reported at endpoint in each of the trials included in this analysis. For instance, the proportion of $30 \%$ responders at endpoint reported in the FM trial included in this analysis was $40.4 \%$ with pregabalin and $30.6 \%$ with placebo. ${ }^{24}$ This is slightly lower than the proportion reported here, presumably as not all patients maintained a $30 \%$ response for the entire 15 weeks of the trial. Similarly, proportions of $30 \%$ responders at endpoint in the SCI ( $42 \%$ pregabalin, $16 \%$ placebo $^{5}$ and $45.7 \%$ pregabalin, $31.4 \%$ placebo $^{23}$ ) and DPN/PHN (59.0\% pregabalin, 37.1\% placebo $^{20}$ and $64.0 \%$ pregabalin, $52.0 \%$ placebo $^{21}$ ) trials were similar to the total proportion after 6 weeks in this analysis.

These data suggest that new proof-of-concept studies could be limited to as little as 3 weeks in duration as this time frame would be sufficient to capture the majority of responders and the most common AEs. For clinical practice, these data indicate that while most patients who will respond do so within the first 3-4 weeks, smaller numbers of new responders continue to emerge at weeks 5 and 6 . Physicians treating patients with therapeutic doses of pregabalin can expect that if a patient will respond, it will likely be within the first 4 weeks; however, in some cases, it may be worthwhile to continue treatment for 6 weeks. This finding is not dissimilar to the reported time to onset for some antidepressants when improvement in depression is the primary endpoint. For example, while most patients who will ultimately respond to fluoxetine treatment do so within the first 2 weeks, approximately $25 \%$ of patients who respond only do so after 4 weeks of treatment. ${ }^{27}$ Thus, if a patient has demonstrated a minimal or partial pattern of response to treatments for pain in the past, it would be important to observe the patient for a full 6 weeks on pregabalin, in order not to miss a pain response that could occur late in treatment.

DPN, PHN, neuropathic pain due to SCI, and FM are distinct diseases with only broad similarities as chronic pain conditions. This analysis looked at each of these conditions as they represent all of the chronic pain conditions for which pregabalin is approved by the US Food and Drug Administration as a treatment option. ${ }^{1}$ As such, this analysis may offer guidance to physicians treating patients with pregabalin as an approved option. At the same time, the emergence of new responders and the majority of common 
AEs within 3-4 weeks in each of the conditions included in this analysis will give greater confidence to researchers planning a proof-of-concept study of pregabalin for another chronic pain condition that they will be able to observe most responses within this time frame.

The IMMPACT (Initiative on Methods, Measurement, and Pain Assessment in Clinical Trials) considerations for improving assay sensitivity in chronic pain clinical trials recommend shorter durations for proof-of-concept studies in order to maximize assay sensitivity. ${ }^{16}$ At the same time, results from a proof-of-concept trial with too short a duration may become a limitation when it comes to designing a longer duration confirmatory trial. ${ }^{16}$ As such, finding the appropriate length for a new proof-of-concept trial in chronic pain can be challenging. Any data that can guide the decision-making process would be of great benefit. These analyses support the use of a 3- to 4-week proof-of-concept trial for pregabalin in chronic pain conditions.

These analyses included pregabalin data only from studies and treatment arms with a flexible dosing design, as this design is a closer representation of the recommended approach in clinical practice where pregabalin should be carefully titrated to the highest tolerable dose. ${ }^{1,17-19}$ The recommended dosing for pregabalin in the USA is up to a maximum of $300 \mathrm{mg} /$ day for DPN, between 300 and $600 \mathrm{mg} /$ day for PHN, up to a maximum of $600 \mathrm{mg} /$ day for SCI, and between 300 and $450 \mathrm{mg} /$ day for FM. ${ }^{1}$ For all conditions, it is recommended that treatment start at $150 \mathrm{mg} /$ day and escalate to $300 \mathrm{mg} /$ day within 1 week based on individual efficacy and tolerability. ${ }^{1}$ Each of the trials in this analysis started with all patients receiving $150 \mathrm{mg}$ /day before escalation to higher doses each week. As such, the emergence of $30 \%$ responders in this analysis after 1 week $(15.0 \%$ with DPN/PHN, 19.0\% with SCI, and 9.6\% with FM) suggests a small degree of effectiveness of pregabalin, in some patients, before the optimal dose is reached. However, not all patients will respond to pregabalin at $150 \mathrm{mg} /$ day, as also indicated here with higher numbers of responders at weeks 2 and 3. Current clinical guidelines support careful escalation to the highest tolerable approved dose, ${ }^{1,17-19}$ with the reported average maintenance dose in the studies included in this analysis being $\geq 370 \mathrm{mg} /$ day.

Consistent with previous studies, ${ }^{19,28}$ these analyses demonstrated that the most common AEs with pregabalin (somnolence and dizziness) typically emerge within the first 2 weeks. Other common AEs in these trials also tended to emerge within the 6 weeks of this analysis. One possible exception was increased weight in patients with FM (with 14 of 39 total instances ${ }^{24}$ emerging in the first 6 weeks). This observation is consistent with previous analyses indicating that increased weight typically emerges following a longer period of pregabalin treatment ( $>56$ days). ${ }^{29}$

The ability to anticipate which AEs may emerge, and when, could potentially improve adherence to pregabalin, by managing patient expectations. Some observational studies of pregabalin have reported lower rates of AEs and this has been credited to ongoing discussions between physicians and their patients regarding potential AEs. ${ }^{30}$ It could be that this is related to a reduction in the patient's anxiety, which then alters how they would perceive the occurrence of a mild AE. ${ }^{19}$

A prior analysis of 31 clinical trials of pregabalin in patients with peripheral neuropathic pain conditions demonstrated that the majority of incidences of some common AEs were transient and resolved prior to the end of their study. ${ }^{19}$ For dizziness and somnolence, respectively, median times to resolution were 9.0 and 14.0 days, with $74.5 \%$ and $64.8 \%$ of incidences resolving prior to the end of the study. ${ }^{19}$ Although it was not a part of the current analysis, this suggests that the majority of cases of somnolence and dizziness would emerge, and then improve or resolve, within the first 4-6 weeks of treatment.

The data in this analysis suggest that the majority of new responders and new AEs emerge within the first 3-4 weeks of treatment with pregabalin. These data could advise researchers planning new proof-of-concept studies of pregabalin in pain conditions and also guide clinical management of patients with DPN, PHN, neuropathic pain due to SCI, and FM.

\section{Acknowlegment}

This study was sponsored by Pfizer. Medical writing support was provided by Joshua Fink of Engage Scientific Solutions, and funded by Pfizer.

\section{Disclosure}

$\mathrm{BP}, \mathrm{BE}$, and $\mathrm{AC}$ are employees of Pfizer and hold stock in Pfizer. The authors report no other conflict of interest in this work.

\section{References}

1. Lyrica ${ }^{\circledR}$ (pregabalin). Prescribing Information. New York, NY, USA: Pfizer Inc; 2011. Available from: http://labeling.pfizer.com/ShowLabeling. aspx?id=561. Accessed September 19, 2014.

2. Davies M, Brophy S, Williams R, Taylor A. The prevalence, severity, and impact of painful diabetic peripheral neuropathy in type 2 diabetes. Diabetes Care. 2006;29(7):1518-1522.

3. Dworkin RH, Portenoy RK. Pain and its persistence in herpes zoster. Pain. 1996;67(2-3):241-251. 
4. Kawai K, Gebremeskel BG, Acosta CJ. Systematic review of incidence and complications of herpes zoster: towards a global perspective. $B M J$ Open. 2014;4(6):e004833.

5. Siddall PJ, McClelland JM, Rutkowski SB, Cousins MJ. A longitudinal study of the prevalence and characteristics of pain in the first 5 years following spinal cord injury. Pain. 2003;103(3):249-257.

6. Wolfe F, Clauw DJ, Fitzcharles MA, et al. The American College of Rheumatology preliminary diagnostic criteria for fibromyalgia and measurement of symptom severity. Arthritis Care Res (Hoboken). 2010; 62(5):600-610.

7. Wolfe F, Smythe HA, Yunus MB, et al. The American College of Rheumatology 1990 criteria for the classification of fibromyalgia. Report of the Multicenter Criteria Committee. Arthritis Rheum. 1990; 33(2):160-172.

8. Wolfe F, Ross K, Anderson J, Russell IJ, Hebert L. The prevalence and characteristics of fibromyalgia in the general population. Arthritis Rheum. 1995;38(1):19-28.

9. Vincent A, Lahr BD, Wolfe F, et al. Prevalence of fibromyalgia: a population-based study in Olmsted County, Minnesota, utilizing the Rochester Epidemiology Project. Arthritis Care Res (Hoboken). 2013;65(5):786-792.

10. Doth AH, Hansson PT, Jensen MP, Taylor RS. The burden of neuropathic pain: a systematic review and meta-analysis of health utilities. Pain. 2010;149(2):338-344.

11. Murray RF, Asghari A, Egorov DD, et al. Impact of spinal cord injury on self-perceived pre- and postmorbid cognitive, emotional and physical functioning. Spinal Cord. 2007;45(6):429-436.

12. Arnold LM, Crofford LJ, Mease PJ, et al. Patient perspectives on the impact of fibromyalgia. Patient Educ Couns. 2008;73(1):114-120.

13. Dworkin RH, O'Connor AB, Audette J, et al. Recommendations for the pharmacological management of neuropathic pain: an overview and literature update. Mayo Clin Proc. 2010;85(3 Suppl):S3-S14.

14. Arnold LM, Clauw DJ, Dunegan LJ, Turk DC. A framework for fibromyalgia management for primary care providers. Mayo Clin Proc. 2012;87(5):488-496.

15. Finnerup NB, Baastrup C. Spinal cord injury pain: mechanisms and management. Curr Pain Headache Rep. 2012;16(3):207-216.

16. Dworkin RH, Turk DC, Peirce-Sandner S, et al. Considerations for improving assay sensitivity in chronic pain clinical trials: IMMPACT recommendations. Pain. 2012;153(6):1148-1158.

17. Attal N, Cruccu G, Baron R, et al. EFNS guidelines on the pharmacological treatment of neuropathic pain: 2010 revision. Eur J Neurol. 2010;17(9):1113-e88.

18. Freeman R, Durso-Decruz E, Emir B. Efficacy, safety, and tolerability of pregabalin treatment for painful diabetic peripheral neuropathy: findings from seven randomized, controlled trials across a range of doses. Diabetes Care. 2008;31(7):1448-1454.
19. Freynhagen R, Serpell M, Emir B, et al. A comprehensive drug safety evaluation of pregabalin in peripheral neuropathic pain. Pain Pract. 2015;15(1):47-57.

20. Freynhagen R, Strojek K, Griesing T, Whalen E, Balkenohl M. Efficacy of pregabalin in neuropathic pain evaluated in a 12-week, randomised, double-blind, multicentre, placebo-controlled trial of flexible- and fixed-dose regimens. Pain. 2005;115(3):254-263.

21. Guan Y, Ding X, Cheng Y, et al. Efficacy of pregabalin for peripheral neuropathic pain: results of an 8-week, flexible-dose, double-blind, placebo-controlled study conducted in China. Clin Ther. 2011;33(2): $159-166$.

22. Siddall PJ, Cousins MJ, Otte A, Griesing T, Chambers R, Murphy TK. Pregabalin in central neuropathic pain associated with spinal cord injury: a placebo-controlled trial. Neurology. 2006;67(10): 1792-1800.

23. Cardenas DD, Nieshoff EC, Suda K, et al. A randomized trial of pregabalin in patients with neuropathic pain due to spinal cord injury. Neurology. 2013;80(6):533-539.

24. Ohta H, Oka H, Usui C, Ohkura M, Suzuki M, Nishioka K. A randomized, double-blind, multicenter, placebo-controlled phase III trial to evaluate the efficacy and safety of pregabalin in Japanese patients with fibromyalgia. Arthritis Res Ther. 2012;14(5):R217.

25. Parsons B, Sanin L, Yang R, Emir B, Juhn M. Efficacy and safety of pregabalin in patients with spinal cord injury: a pooled analysis. Curr Med Res Opin. 2013;29(12):1675-1683.

26. Farrar JT, Young JP Jr, LaMoreaux L, Werth JL, Poole RM. Clinical importance of changes in chronic pain intensity measured on an 11-point numerical pain rating scale. Pain. 2001;94(2):149-158.

27. Nierenberg AA, Farabaugh AH, Alpert JE, et al. Timing of onset of antidepressant response with fluoxetine treatment. Am J Psychiatry. 2000;157(9):1423-1428.

28. Gil-Nagel A, Zaccara G, Baldinetti F, Leon T. Add-on treatment with pregabalin for partial seizures with or without generalisation: pooled data analysis of four randomised placebo-controlled trials. Seizure. 2009;18(3):184-192.

29. Cabrera J, Emir B, Dills D, Kevin Murphy T, Whalen E, Clair A. Characterizing and understanding body weight patterns in patients treated with pregabalin. Curr Med Res Opin. 2012;28(6):1027-1037.

30. Toelle T, Varvara R, Nimour M, Emir B, Brasser M. Pregabalin in neuropathic pain related to DPN, cancer and back pain: analysis of a 6-week observational study. Open Pain J. 2012;5:1-11.
Journal of Pain Research

\section{Publish your work in this journal}

The Journal of Pain Research is an international, peer-reviewed, open access, online journal that welcomes laboratory and clinical findings in the fields of pain research and the prevention and management of pain. Original research, reviews, symposium reports, hypothesis formation and commentaries are all considered for publication.

\section{Dovepress}

The manuscript management system is completely online and includes a very quick and fair peer-review system, which is all easy to use. Visit http://www.dovepress.com/testimonials.php to read real quotes from published authors. 